\title{
NEARLY ISOTROPIC 6-UPU PARALLEL MANIPULATOR
}

\author{
A. Chandrashekhar \\ Asst. Prof, Mechanical Engineering Department, \\ The ICFAI Foundation for Higher Education, Hyderabad, India \\ G. Satish Babu \\ Professor, Department of Mechanical Engineering, \\ JNTUH College of Engineering Hyderabad, INDIA
}

\begin{abstract}
In this paper condition number is utilized as a measure to distinguish ideal structures in a parallel robot. A few structures have been examined in a 6 UPU robot with a specific end goal to recognize most ideal setups. This technique has driven our exploration examination to recognize isotropic or almost isotropic designs. Matlab code is produced to get results which have been spoken to in a graphical structure.
\end{abstract}

Keywords: Performance Index, Isotropic Configurations, Condition Number

Cite this Article: A. Chandrashekhar and G. Satish Babu, Nearly Isotropic 6Upu Parallel Manipulator. International Journal of Design and Manufacturing Technology 6(2), 2015, pp. 54-60.

https://iaeme.com/Home/issue/IJDMT?Volume $=6 \&$ Issue $=2$

\section{INTRODUCTION}

Salisbury and Craig (1) are the first to apply the condition number thought to frameworks. They have used the condition number of the Jacobian Matrix as an improvement measure to get impeccable estimations for the frameworks with the two revolute joints and Stanford JPL Articulated hand.

Tanev and Stoyanov (2) connected the execution lists, the skill record, manipulability, condition number and least particular worth to a SCARA sort robot and think about the lists Kim and Tsai (3) displayed a parallel controller to accomplish translational movement of the moving stage. It was demonstrated that the turning incitation system results in numerous solitary focuses with in the workspace. Because of the orthogonal plan of the appendages, the Jacobian network is constantly isotropic and the controller carries on like a customary X-Y-Z Cartesian machine.

Mayorga et al (4) exhibited a straightforward execution file for robot controller kinematic plan advancement and best stance determination. This record is gotten from 
a homogenized isotropy state of an appropriately weighted Jacobian network. The proposed file is connected to the kinematic outline advancement and best stance determination of a repetitive controller.

Kucuk and Bingul (5) contemplated manipulability measure and condition number for the ideal robot plan. The structures of the robot controllers were thought about in light of the basic length record and worldwide molding list.

In this paper 6 UPU robot has been studied in order to identify optimum configurations based on condition number.

\section{MEASURE OF KINEMATIC PERFORMANCE}

\subsection{Condition number}

To optimize Let us consider the following system:

$$
A x=b
$$

Where $\mathrm{A}$ is an $\mathrm{n} x \mathrm{n}$ matrix and $\mathrm{x}$ and $\mathrm{b}$ are $\mathrm{n}$-dimensional vectors. The condition number of the matrix $\mathrm{A}$ is a measure of its natural resistance to round-off error when the solution of the linear system is computed. This resistance is expressed by the application factor by which a relative error $\frac{\|\delta b\|}{\|b\|}$ In the data is multiplied to lead to a relative error $\frac{\|\delta x\|}{\|x\|}$ In the solution and it is called the condition number of the matrix.

$$
\begin{aligned}
& \frac{\|\delta x\|}{\|x\|} \leq k \frac{\|\delta b\|}{\|b\|} \\
& k=\|A\|\left\|A^{-1}\right\|
\end{aligned}
$$

Where

Is the condition number of $\mathrm{A}$. which defines an upper bound for the amplification of the relative error. This definition of the condition nuber can be used with different matrix norms. The Euclidean or frobenius-norm was used, which is defined as:

$$
\|A\|=\sqrt{\operatorname{tr}\left(\mathrm{AWA}^{T}\right)}
$$

Where $\mathrm{W}$ is the weight matrix

$W=\frac{1}{n} I$ and $\mathrm{A}$ is assumed to be $\mathrm{n} x \mathrm{n}$ or equivalently

$$
\|A\|=\sqrt{\sum_{i=1}^{i=m} \sum_{j=1}^{j=n}\left|a_{i j}\right|^{2}}
$$


Other definitions for the norm could be adopted. For instance, the square root of the largest Eigen value of $\mathrm{A}^{\mathrm{T}} \mathrm{A}$ is often used. This definition has the advantage of being applicable to non square matrix. When this definition is adopted, the condition number of a matrix A becomes the square root of the ratio of the largest to the smallest Eigen value of the matrix $\mathrm{A}^{\mathrm{T}} \mathrm{A}$. the Euclidean norm was used here because it is framedinvarient and it is also very easy to compute.

The condition number of A Jacobean matrix of a manipulator is

$$
k(J)=k\left(J^{-1}\right)=\left\|J^{-1}\right\|\|J\|
$$

\section{6-UPU PARALLEL MANIPULATOR}

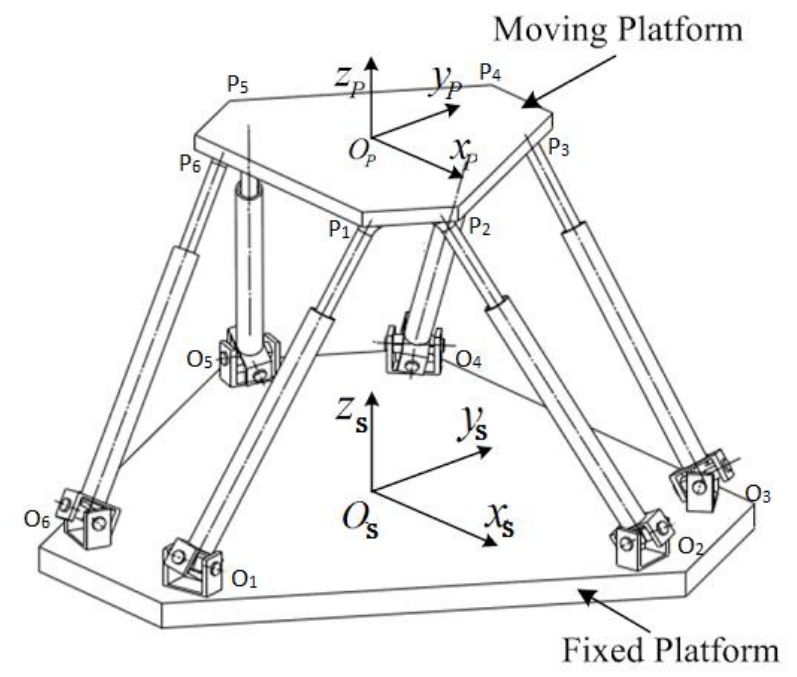

Figure 1 Kinematic model of 6-UPU Parallel manipulator

The 6 UPU parallel manipulator shown in fig. 1 consists of a fixed platform and moving platform connected by six identical limbs. The reference frame attached to the fixed platform is $\mathrm{O}_{\mathrm{s}}-\mathrm{x}_{\mathrm{s}}, \mathrm{y}_{\mathrm{s}}, \mathrm{Z}_{\mathrm{s}}$. and the frame $\mathrm{O}_{\mathrm{p}}-\mathrm{x}_{\mathrm{p}}, \mathrm{y}_{\mathrm{p}}, \mathrm{Z}_{\mathrm{p}}$ is attached to the the moving platform. The distances between the joints in moving and fixed platforms are:

Fixed Platform:

$$
\begin{aligned}
& \mathrm{O}_{1} \mathrm{O}_{2}=\mathrm{O}_{3} \mathrm{O}_{4}=\mathrm{O}_{5} \mathrm{O}_{6}=\mathrm{a}_{1} \\
& \mathrm{O}_{2} \mathrm{O}_{3}=\mathrm{O}_{4} \mathrm{O}_{5}=\mathrm{O}_{6} \mathrm{O}_{1}=\mathrm{a}_{2}
\end{aligned}
$$

Moving Platform:

$$
\begin{aligned}
& \mathrm{P}_{1} \mathrm{P}_{2}=\mathrm{P}_{3} \mathrm{P}_{4}=\mathrm{P}_{5} \mathrm{P}_{6}=\mathrm{b}_{1} \\
& \mathrm{P}_{2} \mathrm{P}_{3}=\mathrm{P}_{4} \mathrm{P}_{5}=\mathrm{P}_{6} \mathrm{P}_{1}=\mathrm{b}_{2}
\end{aligned}
$$

\section{OPTIMAL DESIGN OF 6-UPU PARALLEL MANIPULATOR USING PERFORMANCE INDICES}

\subsection{Jacobian Matrix}

In this paper an $m \times n$ dimensional homogenous Jacobian matrix mapping the actuator velocities to the independent end effect velocities is considered.

Consider the following general equation.

$q=J x$ 
Where $\dot{q}$ is an $m$-dimensional vector containing the $m$ actuator velocities, $\dot{x}$ the $n$ - dimensional vector of end effect velocities, and $J$ is the $m \times n$ Jacobian matrix. For the 6 UPU manipulator

$$
J=\left(\begin{array}{ccc}
\hat{K}_{1}^{T} & \ldots & \left({ }^{p} R_{s} w_{1} \times \hat{K}_{1}\right)^{T} \\
\vdots & \ddots & \vdots \\
\hat{K}_{6}^{T} & \cdots & \left({ }^{p} R_{s} w_{6} \times \hat{K}_{6}\right)^{T}
\end{array}\right)
$$

Where $\hat{K}_{i}$ denotes a unit vector along the $i$ th active prismatic joint pointing from its joint at the moving platform. The platform-attached and the base-attached coordinate systems are referred to by the letter $s$ to $p$, respectively, Fig-. Accordingly, ${ }^{p} R_{s}$ is the rotation matrix from $s$ to $p$, and $w_{i}$ is the constant position vector of the $i^{\text {th }}$ joint in $q$, Fig.1.

$$
q=\left(d_{1} d_{2} \ldots \ldots . d_{6}\right)
$$

The instantaneous twist, $\$_{P}$, of the moving platform can be expressed as

$\$_{P}=\hat{\theta}_{1} \hat{\$}_{1, i}+\hat{\theta}_{2} \hat{\$}_{2, i}+\hat{d}_{3} \hat{\$}_{3, i}+\hat{\theta}_{4} \hat{\$}_{4, i}+\hat{\theta}_{5} \hat{\$}_{5, i}$

for $i=1,2, \ldots . .5$

where

$$
\begin{aligned}
& \hat{\$}_{1, i}=\left(\begin{array}{l}
s_{1, i} \\
\left(b_{i}-d_{i}\right) \times s_{1, i}
\end{array}\right) \\
& \hat{\$}_{2, i}=\left(\begin{array}{l}
s_{2, i} \\
\left(b_{i}-d_{i}\right) \times s_{2, i}
\end{array}\right) \\
& \hat{\$}_{3, i}=\left(\begin{array}{l}
0 \\
s_{3, i}
\end{array}\right) \\
& \hat{\$}_{4, i}=\left(\begin{array}{l}
s_{4, i} \\
b_{i} \times s_{4, i}
\end{array}\right) \\
& \hat{\$}_{5, i}=\left(\begin{array}{l}
s_{5, i} \\
b_{i} \times s_{5, i}
\end{array}\right)
\end{aligned}
$$

Where $\mathrm{s}_{\mathrm{j}, \mathrm{i}}$ is a unit vector along the $\mathrm{j}^{\text {th }}$ joint axis of the $\mathrm{i}^{\text {th }} \operatorname{limb}, b_{i}=\overline{P B}_{i}$ and $d_{i}=\bar{A}_{i} B_{i}=d_{3, i} S_{3, i}$

The reciprocal screw, denoted as $\hat{\$}_{r, 1, i}$ is pointing in a direction perpendicular to the joint axes of the universal joint. 


$$
\begin{aligned}
& \hat{\$}_{r, 1, i}=\left(\begin{array}{l}
0 \\
n_{i}
\end{array}\right) \\
& \hat{\$}_{r, 1, i}^{T}=0 \\
& J_{C} \$_{P}=0
\end{aligned}
$$

Where

$$
J=\left(\begin{array}{ll}
n_{1}^{T} & 0_{1 \times 3} \\
n_{2}{ }^{T} & 0_{1 \times 3} \\
n_{3}{ }^{T} & 0_{1 \times 3} \\
\vdots & \vdots \\
n_{6}{ }^{T} & 0_{1 \times 3}
\end{array}\right)
$$

Reciprocal to all passive joint screws of the ith limb can be identified as zero pitch screw along the line passing through the two universal joints

$$
\begin{aligned}
& \hat{\$}_{r, 2, i}=\left(\begin{array}{l}
s_{3, i} \\
b_{i} \times s_{3 \times i}
\end{array}\right) \\
& \hat{\$}_{r, 2, i}^{T} \$_{P}=d_{3, i} \\
& J_{x} \$_{P}=q
\end{aligned}
$$

Where

$$
\begin{aligned}
& J_{x}=\left(\begin{array}{ll}
\left(b_{1} \times s_{3,1}\right)^{T} & s^{T}{ }_{3,1} \\
\left(b_{2} \times s_{3,2}\right)^{T} & s_{3,2}^{T} \\
\left(b_{3} \times s_{3,3}\right)^{T} & s^{T}{ }_{3,3} \\
\vdots & \vdots \\
\left(b_{6} \times s_{3,6}\right)^{T} & s_{3,6}^{T}
\end{array}\right) \\
& q=\left[d_{3,1}, d_{3,2}, d_{3,3}, d_{3,4}, d_{3,5}, d_{3,6}\right]^{T} \\
& \text { Therefore } q_{0}=J \$_{p}
\end{aligned}
$$

Where

$$
J=\left(\begin{array}{ll}
\left(b_{1} \times s_{3,1}\right)^{T} & s_{3,1}^{T} \\
\vdots & \vdots \\
\left(b_{6} \times s_{3,6}\right)^{T} & s_{3,6}^{T} \\
n_{1}^{T} & 0_{1 \times 3} \\
\vdots & \vdots \\
n_{6}^{T} & 0_{1 \times 3}
\end{array}\right)
$$




\section{RESULTS}

Using Matlab the following results have been obtained for 6 UPU spatial manipulators. The results regarding optimum structures in which nearly isotropic configurations were identified have been presented in this section.

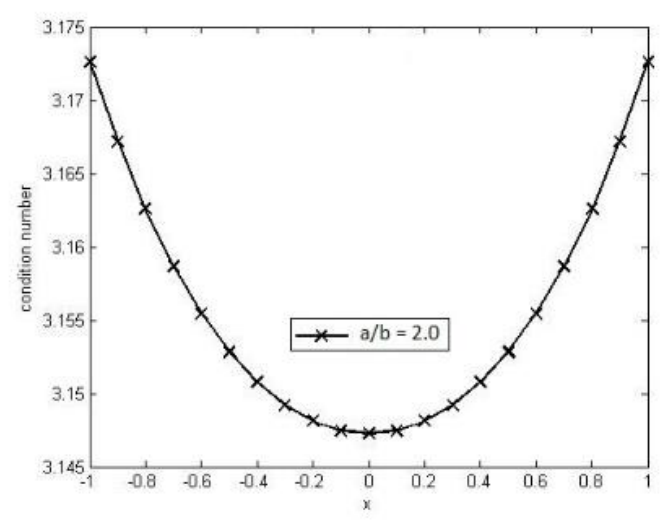

Figure 2 Graph illustrating condition number vs Reach $(\mathrm{a} / \mathrm{b}=2.0)$

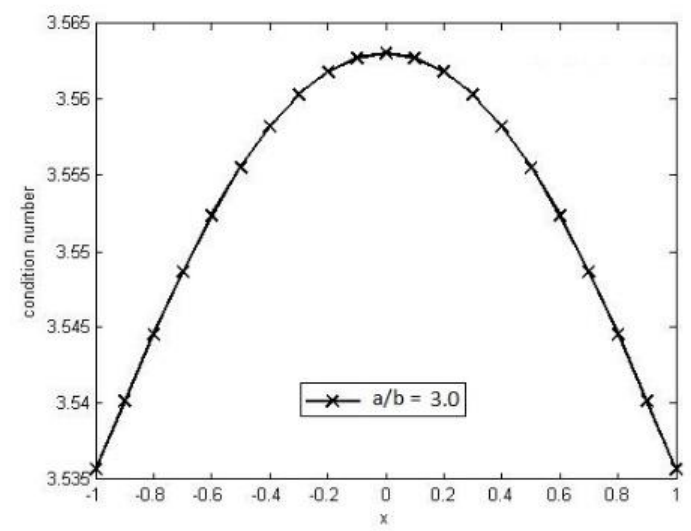

Figure 3 Graph illustrating condition number vs Reach $(\mathrm{a} / \mathrm{b}=3.0)$

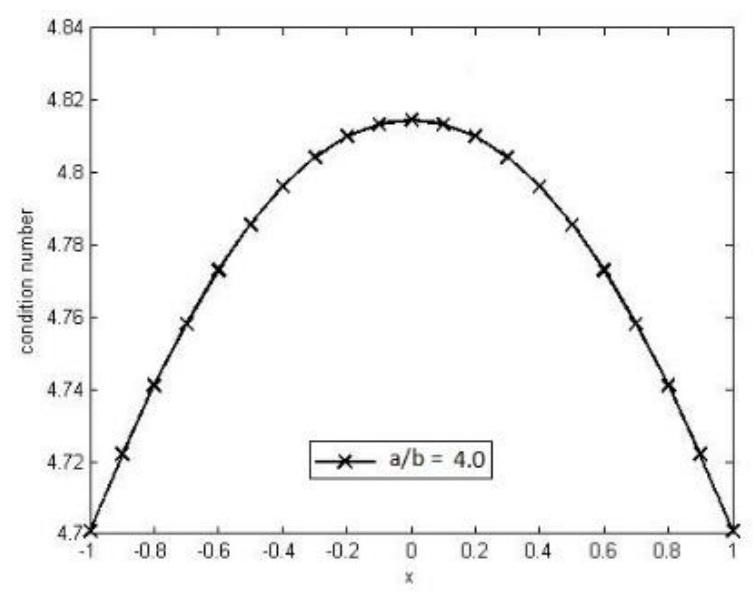

Figure 4 Graph illustrating condition number vs Reach $(\mathrm{a} / \mathrm{b}=4.0)$ 


\section{CONCLUSION}

Using condition number as a performance index, several structures have been analyzed in 6 UPU robot in order to identify the nearly isotropic configurations. Finally in three structures nearly isotropic configurations have been found.

\section{REFERENCES}

[1] J. Kenneth Salisbury and John J. Craig. "Articulated Hands: Force Control and Kinematic Issues," The international Journal of Robotics Research, Vol.1, No. 1, pp 4-17, spring 1982.

[2] Tanio Tanev and Bogdan Stoyanov. "On the performance Indexes for Robot Manipulators," Journal of Bulgarian Academy of sciences, Problems of Engineering Cybernetics and Robotics 49, pp 64-71 (2000).

[3] Han Sung Kim and Lung-Wen Tsai. "Design Optimization of a Cartesian Parallel Manipulator,” ASME the Journal of Mechanical Design, Vol. 125, pp 43-56, March 2003.

[4] Rene V. Mayorga et al. "A kinematics performance index based on the rate of change of a standard isotropy condition for robot design optimization," Robotics Autonomous Systems 53, pp 153-163 (2005).

[5] Serdar Kucuk and Zafer Bingul. "Comparitive study of performance indices for fundamental robot manipulators," Robotics Autonomous Systems 54, pp 567-573 (2006).

[6] A. Chandrashekhar and G. Satish Babu, Force Isotropy of Three-Limb Spatial Parallel Manipulator. International Journal of Mechanical and Engineering Technology 6(6), 2015, pp. 01-08. 